\title{
Intelligent Transportation System (ITS) for Smart-Cities using Mamdani Fuzzy Inference System
}

\author{
${ }^{1}$ Kashif Iqbal \\ Government College University \\ Lahore, Pakistan
}

\author{
${ }^{2}$ Muhammad Adnan Khan, \\ Sagheer Abbas, Zahid Hasan \\ School of Computer Science \\ National College of Business \\ Administration \& Economics, \\ Lahore, Pakistan
}

\author{
${ }^{3}$ Areej Fatima \\ Department of Computer Science \\ Lahore Garrison University, \\ Lahore, Pakistan
}

\begin{abstract}
It is estimated that more than half of the world population lives in cities according to (UN forecasts, 2014), so cities are vital. Cities, as we all know facing with complex challenges - for smart cities the outdated traditional planning of transportation, environmental contamination, finance management and security observations are not adequate. The developing framework for smart-city requires sound infrastructure, latest current technology adoption. Modern cities are facing pressures associated with urbanization and globalization to improve quality-of-life of their citizens. A framework model that enables the integration of cloud-data, social network (SN) services and smart sensors in the context of smart cities is proposed. A service-oriented radical framework enables the retrieval and analysis of big data sets stemming from Social Networking (SN) sites and integrated smart sensors collecting data streams for smart cities. Smart cities' understanding is a broad concept transportation sector focused in this article. Fuzzification is shown to be a capable mathematical approach for modelling traffic and transportation processes. To solve various traffic and transportation problems a detailed analysis of fuzzy logic systems is developed. This paper presents an analysis of the results achieved using Mamdani Fuzzy Inference System to model complex traffic processes. These results are verified using MATLAB simulation.
\end{abstract}

Keywords-Information Communication Technology (ICT); Internet of Things (IoT); Intelligent Transportation System (ITS); Fuzzy Inference System (MFIS); Traffic Congestion Conditions (TCC); SNA; MF; Mamdani Fuzzy Inference System (MFIS)

\section{INTRODUCTION}

It is the time of Social Networking, Cloud Computing and explosion of smart sensors deployed everywhere [1]. According to UN survey in 2014, more than half of world's population now living in urban areas [2] and increasing surely alerting city planners. Connected cities emerge when Internet of Things (IoT) technologies and socially-aware network systems aggregate administrations over a whole connected metropolitan territory. When thinking of connected urban areas, one may think of high tech cities that have the prominent cutting-edge technologies for their citizens like Copenhagen, London, New York, Chicago, Stockholm or Amsterdam. However, small residential communities have also been benefiting from interfacing individuals, administrations, city infrastructure and services. This article investigates city transportation problem and a portion of the difficulties that are involved with developing widespread IoT techniques. The coalition of world-class IoT improvement anticipates working with each of these smart urban communities that enable citizens to make technology utilization more sensible, adaptable and sustainable. Many urban cities and towns around the globe are turning to socially connected smart devices to solve urban problems [3], for example, traffic congestion, environmental contamination, healthcare, security surveillance to enhance the living standards for their general public everyday comforts. Smart sensors that are installed throughout the city, in vehicles, in buildings, in roadways, in control monitoring systems, security surveillance and applications and devices that are utilised by individuals who are living or working in the city [4]. Delivering information to the public that is utilizing through these high tech smart cities opportunities. The bigdata analytics utilized to decide on how public spaces are planned, how to make the best utilization of their assets and how to convey administrative notifications more proficiently, viable and appropriately [5].

Therefore, most urban cities have embraced huge investments during recent decades in Information Communication Technology (ICT) infrastructure including computers, broadband availability and some sensing frameworks [6]. These infrastructures have engaged various inventive administrations in territories, for example, demographic sensing, urban coordination and real information that makes living ones close. Such administrations have been widely sent in a few urban cities, accordingly exhibiting the potential advantages of ICT frameworks for organisations and the natives themselves [7]. During most recent years it has

additionally seen a blast of sensor distribution, along with the development of adaptive systems, internet-of-things [8] current advancements of sensor-based systems have emerged. Currently, the advantages of social communication and internet-of-things distributions for smart urban areas have likewise been exhibited [9].

Current Smart City data analysis implies complex stream analytics for a comprehensive set of activities aiming to turn into real actionable outcomes [10]. The analysis comprises of following contributions: 
1) Analysis of thousands of traffic blockage cases, road capacity measures, traffic signalling and dynamic, consistent information to give a better message to the citizens.

2) Events, episodic road examination, utilising real-data gathered by citizens, devices and sensors.

3) Turning web-based into social media information, important city events analysis, assumptions, examinations, and numerous other things. Consolidating information from physical (sensors/devices) and social sources (social organisations) can give full, essential information and adds to better assessment and bits of knowledge.

Over-all speaking, smart cities realization is a broad concept so, the transportation sector is focused in this article. Fuzzy logic is one of the strongest candidate solution for mathematical based modelling. In this article fuzzy logicbased solution is proposed for transportation problem. The input parameters are: Vehicular Speed (VS), Road Capacity (RC), Traffic Signals (TS), Trip Riding Distance (RD) and Distance Traffic Signals (DTS). A detailed Transportation fuzzy logic system is developed based on rule-based inferencing to solve the traffic congestion issues. Analysis of the results obtained using Mamdani Fuzzy Inference System is verified using MATLAB Simulation

The objective of this paper is to analysis key issues and the solutions about traffic congestion in a smart city in the light of critical inducing aspects. The rest of the paper is structured as follows: Section II gives an overview of related and similar works that can be found in the international literature. Section III presents the fundamental architecture and approach. Sections IV and V presents technical details, sentiment analysis of problems and a conceptual model for smart-cities. Section VI provides a proposed Mamdani Fuzzy Inference System (MFIS) based results analyses, the work is planned in the context of simulation and Section VII contains conclusion and future work to be planned in the context of smart drive mobile apps.

\section{LITERATURE REVIEW}

A smart IoT system which automatically notifies necessary information of passengers after triggering of shock detector sensors to lowering loss rates in accidents and alert nearby local public safety organization about the physical location of accident suggested by Nasr et al. [11]. Rizwan et al. industrialize a smart traffic management system roadside unit. It carries alternate routing to avoid traffic blocking and increase traffic flow through IoT and lower traffic density, offers predictive analytic technique (Big-data techniques) [12]. Scalable Enhanced Road Side Unit, SERSU, proposed by Al-Dweik et al. used wireless communication network and radio frequency adaptive traffic control system, pollution detection system and weather information system. SERSU components were placed on the roadsides with various breaks, capturing generated sensor signals by vehicle sensors module [13]. Modern techniques in cars, internet and their current and future relationship, detail history of usage of electronic devices in automobiles, and social implication of these technologies briefly studied by Goggin [14]. Joshi et al. made infrared-based sensor system, which to monitor traffic flow and provides alternate road traffic routing path to drivers for the avoidance of traffic crowding capture infrared radiations emitted by vehicles on road surface [15]. Handte et al. designed IoT enabled the navigational system for real transport facility, provided complete guidance of routes to bus riding passengers for urban bus riders in Madrid, which were assisting in micro-navigation, expects massive aware routes. A system to communicate with onboard sensors to sense the presence of onboard passengers, this system was based on mobile devices. Their system collected real-world bus user's response for better accessibility of travel information [16]. Zanella et al. advised web-based service approach for IoT service architecture to resolve integration issues for different end node devices connected to IoT system Zanella et al. also evaluated key ideas, facilities and solution are currently available for implementation of IoT based smart cities [4]. Technological challenges and socio-economic opportunities in developing and designing of future smart cites discussed key by Theodoridis et al., they also suggested 3-tier IoT nodes and 3-plane architecture model. Further, they develop a city scale test bed for future internet and IoT experimentation [17]. A hierarchy which combines smart homes and smart cities described by Skouby et al., they also proposed a four-layered model to join end nodes IoT devices, communication technologies like distributed artificial intelligence and cloud of things [6]. Gubbi et al. presented Radio Frequency Identification (RFID's) a user-centric cloud-based vision of implementation of IoT, by the interaction of public and private clouds, major research trends, IoT application domain, current and future enabling technologies etc. that will drive IoT shortly [14]. Base Station arrangement, based architecture sensor system for intelligent traffic light system (TLS) suggested by Chong et al. They designed intelligent software, implemented on TLS which continuously communicates with the base station and calculates green light time, and provide monitoring of traffic by officers [18].

Internet of Vehicle (IoV), a unique solution for smart traffic management is discussed by Dandala et al. They argued that IoV can be an effective solution conventional IoT based traffic management technique to overcome traditional traffic issues. Further, they described to be a reality which is a vehicle to vehicle's owner that IoV needs four types of communication, a vehicle to vehicle, a vehicle to centralize server and vehicle to the third party like police patrol, ambulance, etc. [19]. Cognition was used for user authentication in vehicles [26]. Sagheer et al. proposed a fuzzy inference system to avoid traffic congestion using bioinspired method [27].

Density-based signalling to overwhelmed issues raised by fixed time signalling for example in fixed time signalling method the traffic lights have predefined periodic time system suggested by Thakur et al. provides intelligent signalling by assigning the greener signal to dense traffic region to avoid congestion by continuously evaluating traffic density [20].

Ramchandra et al. proposed a comparable system which device traffic lights by using average speed of vehicles dynamically according to the density of traffic. In this proposed system every vehicle is equipped with On-Board 
Device (OBD) distribute data to centralise server using Zigbee protocol which acquires vehicle speed data process [21].

Chowdhury et al. proposed intelligent traffic light system for messaging between emergency vehicles infrastructure and to reduce traffic congestion and increase reliability to traffic signals. The proposed system considers the priority of vehicle depends on the type of incident and to secure signals from hacking [22]. Some shortcomings in the traditional intelligent transportation system and argued to prefer Radio Frequency Identification (RFID) pointed out by Ou et al., sensor system and networking technologies to overwhelmed traditional intelligent transportation systems [23].

Information-Centric Networking to project and device Future Internet Architecture proposed by Amadeo et al. In Information-Centric Networking which uses IoT submissions to access data of every end node device having unique location name [24].

\section{SMART-City Key FEATURES REALIZATION}

The availability of smart solutions for cities has risen quickly over the most recent years. Therefore, technical solutions exist for each city to become smarter. The challenge today is mostly to execute proper solutions proficiently, as opposed to just concentrating on innovations. Smart city areas cannot be developed through a patchwork approach, yet by the well-ordered adoption of incremental changes. The most proper way of smart-city realisation is introducing a smart system working group of volunteers characterize its manageability vision and afterwards lays out an electronic well-ordered guide and execution design. The capacity to distinguish the acutest bottlenecks to send coordinated and flexible solutions and afterwards to use these outcomes into other smart community's activities requires involvement and strong specialised expertise.

Smart City Key Resources: Transportation, Climate Change, Energy, Utilities, Security Surveillance, Healthcare, Business Management, etc.

Connected cities enhance the experience of workers by analyzing data and smart city coordinators by breaking down information from reporting frameworks including sensors, roadside cameras, brilliant monitoring systems and speed check signs. Applying IoT innovations to solve urban community's issues includes gathering the information that is collecting from sensors, recordings by cameras, interpersonal organizations and brilliant devices that are examining realdata. This data is delivered noteworthy bits of knowledge that are utilized straightforwardly to trigger actuators that are associated with smart devices. For example, versatile smart city assets, connected by implications, to illuminate choices on policy and to streamline jobs. In smart urban communities, these arrangements include monitoring geographic information from Global Positing System (GPS) trackers and RFID labels on vehicles [15], structures, buildings and power stations, breaking down the proceed of vehicles to recognize occurrences or blockages. Smart buildings security, interpersonal organizations, city administrations are straightforwardly modifying frameworks continuously to control the activity stream in city events, security observation investigation and reduce traffic delays. Authentic analysis of city traffic, security investigation and movement blockage and roadside sensors information can likewise be utilized to alter time delays, misinterpreting security observation, speed cutoff points and city toll tax, control security monitoring and activity stream in the more flows for long-term outcomes. To route movement around incidents, sensors additionally write about the state of streets conditions, weather updates, buildings structures, road lights and extensions with the goal that support to schedule maintenance when required.

Smart cities will make emerging activities in transportation, utilities, smart buildings and smart security. Smart city design plan leaders shaped a working group of ecosystem system accomplices to evaluate robust city community's abilities and guide a long-term vision that coordinates with the city's future planning. Smart city planners have endorsed digitalising citywide assets like fast travel framework, smart buildings, smart security, electric transport and is additionally pushing ahead for far-reaching IoT hub that will pioneer digital city infrastructure.

Designing a roadmap for smart-cities is based on four core pillars: Connectivity is the foundational layer of a smart-city. In real-time data is collected about peoples, places and things by smart sensors and this data are stored on cloud application servers to analyze and utilised to take better real-time decisions and planning as shown in Fig. 1.

Mobility means moving peoples, goods and information efficiently and efficiently. The economic-mobility means regardless of circumstances online job seekers in smart-cities find maximum jobs available that are not handy via public transportation.

Next is security improving public and private places security, data protection and cyber-security while using latest ICT's technologies on-line and off-line.

Sustainability, of course, is focusing on sustainable practices in critical sectors of cities such as transportation, energy consumption, climate change, utilities, security observations, and financial services.

Implementation of smart-cities solutions may have three things every day for their citizen, i.e., creates values, generates revenues and cut costs depending on value exchange smart systems and smart projects.

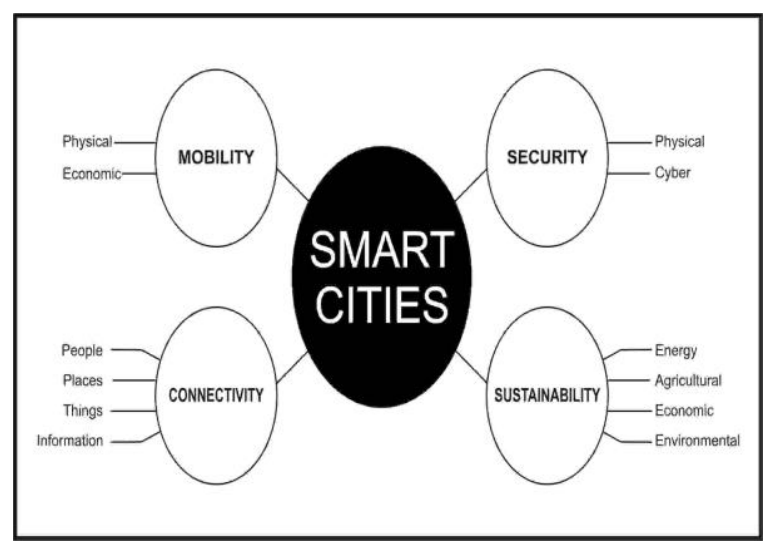

Fig. 1. Road-map four pillars of a smart-city. 


\section{A. Smart Cities Framework Model Overview}

The adaptive data analysis stage is plotted out in the background. It is made out of different layers, bring down a level (devices, communication planes), middle layers (data, information examination) at higher layers (application, dashboard planes). At each layer, distinctive programming code chunks perform specific operations, related to collecting data, messaging, data accessing, semantic annotation, examination or perception where applications can join segments from different layers in light of their specific prerequisites. Along these advances toward getting to be plug and play and can be mainly used in smart-city sectors applications. The present extraordinary cutting-edge advances of portability smart-phones, interpersonal organization services and objects are coordinated together for a new time machine to machine and person to person communication correspondence [17].

\section{1) Main Components of the Model}

The layered framework model of a smart city as shown in Fig. 2 is having four main layers described below as:

\section{a) Sensing Layer}

Sensor Layer comprises tens to thousands of sensor hubs connected using smart remote technologies. They gather data from the environment and convey it to other connected devices that pass the data to the cloud server over the Internet.

\section{b) Communication Layer}

Wireless heart innovative technology gives excellent remote protocol access to the full range of processors capacity, control, and resource management to applications. DigiMesh is an exclusive shared systems networking topology for use in remote end-point network connectivity through the physical Internet.

\section{c) Data Layer}

The capacity and processing of data should be possible on the edge of the networks itself or in a cloud server. If any preprocessing of data is a need, then it is typically done at either the sensor or some other proximate device.

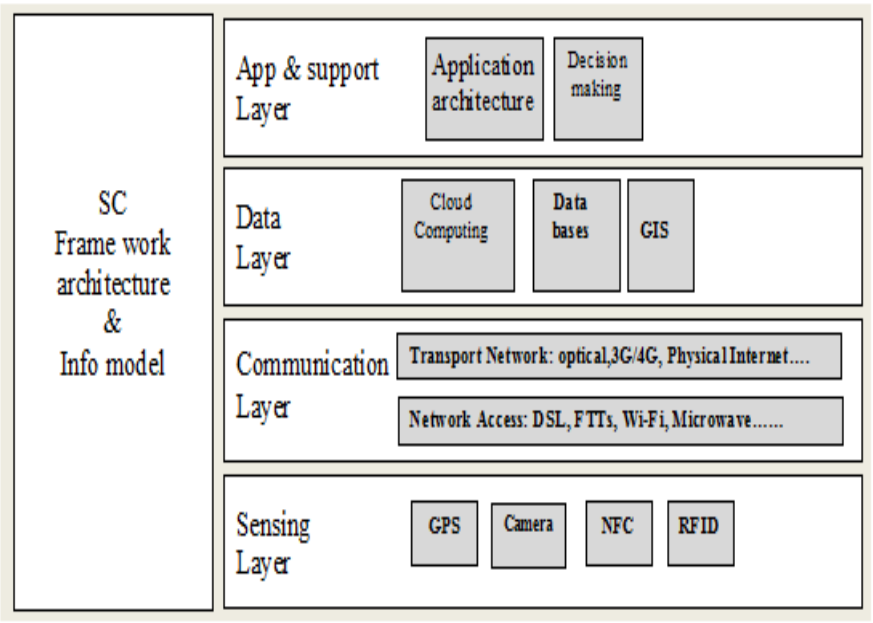

Fig. 2. Layered Model of a smart-city.
The processed data is then regularly sent to a remote server. The capacity and processing abilities of an IoT object are additionally controlled by the assets accessible, which are regularly exceptionally compelled because of constraints of capacity, vitality, control, and computational ability.

\section{d) Application Layer}

The application layer is responsible for data organization and presentation. The application layer on the Internet is regularly in light of Hyper Text Transfer Protocol (HTTP)/ File Transfer Protocol (FTP) standards. The proposed events in this examination are sharing of dynamic data to customers using mobile phones as a particular device. It may be HTTP is not reasonable in resource enabling situations since it is relatively verbose and this manner brings about a significant parsing overhead. Many other innovative conventions have been produced for IoT resources, for example, Message Queue Telemetry Transport (MQTT) and Constrained Application Protocol (CoAP).

Along with these four layers following components move toward becoming plug and play real-time integration and stream-analytics that can be utilized explicitly by specific smart applications framework by adaption of these technical modules given below:

\section{a) Data Wrapper}

It is a program that extracts the content from a particular information source and translates it into an organization format. Using sensory meta-data, it extends a generic way to describe features of sensors, about the data stream that containing general information. A semantic annotation module annotates the sensory parsed data.

\section{b) Data Aggregation}

For data aggregation, the source information originates from public records online databases. The information is packaged into aggregate reports this information is useful for business, marketing, local and government organizations. It reduces the large volume of data, i.e. the size of raw sensory observations delivered by the data wrappers by using data compression techniques and time series analysis.

\section{c) Data Federation}

Answers to user queries, according to the requirements it first finds the relevant stream. It then translates the user request into Resource Description Framework-Stream Processing (RDF-RSP) queries and obtains results accordingly. As fast changing real-world data from sensors and online services evolves IoT-based smart environment monitoring, real-time processing and analytics based on RSP semantics. RDF query language manages continuous data streams SPARQL, and CQELS languages support RDF reasoning.

\section{d) Event Detection}

The event detection is the identification of items, events and observations, i.e. constraints on what defines an event is relaxed or usually modelled as a set of thresholds or probabilities. In city sectors, it provides tools or web software's applications that monitor urban areas events such as the need for clearing transport deadlock, emergency 
facilities, irrigation facilities, pest identification in crops and growing view of the traffic congestion form real-time annotated and aggregated data streams.

\section{CONCEPTUAL FRAMEWORK FOR SMART-CITIES}

The proposed technique in this study is sharing of dynamic information to users using smartphones as a communication device. Current day advanced modern technologies of knowledge mobilization, cloud-servers and the smart-city app are integrated into a new era of quick communication. Advanced smart-phones may have limited sensing capabilities but enhanced computational strength, lesser cost, excessive usage, availability of Global System for Mobile communication (GSM) and mobile internet signals, availability of different sensors in smartphones like a gyroscope, digital compass, proximity sensor, etc. Services available like Google map, Google weather, IBM live streaming analytics etc. is prime motivation to use a smartphone as sensor I/O device in the proposed system. Moreover, specialised and more accurate sensors like accelerometer, Global Positioning System (GPS), and shock sensor etc. services are realised. Also, specific and more accurate sensors like accelerometer, global positioning system, and amaze sensor so on so forth are outlined and created on various stages and new technologies integrating with existing technologies in a single integrated system that is beyond the scope of the current proposes a study as shown in Fig. 3.

The proposed system aims to provide efficient and effective smart cities traffic infrastructure. In this study, we show the concepts of cloud computing, bid-data analysis, internet of things, human-computer interactions, software engineering paradigm etc. can be the realization of smartcities traffic framework to improve the living standards of their citizens.

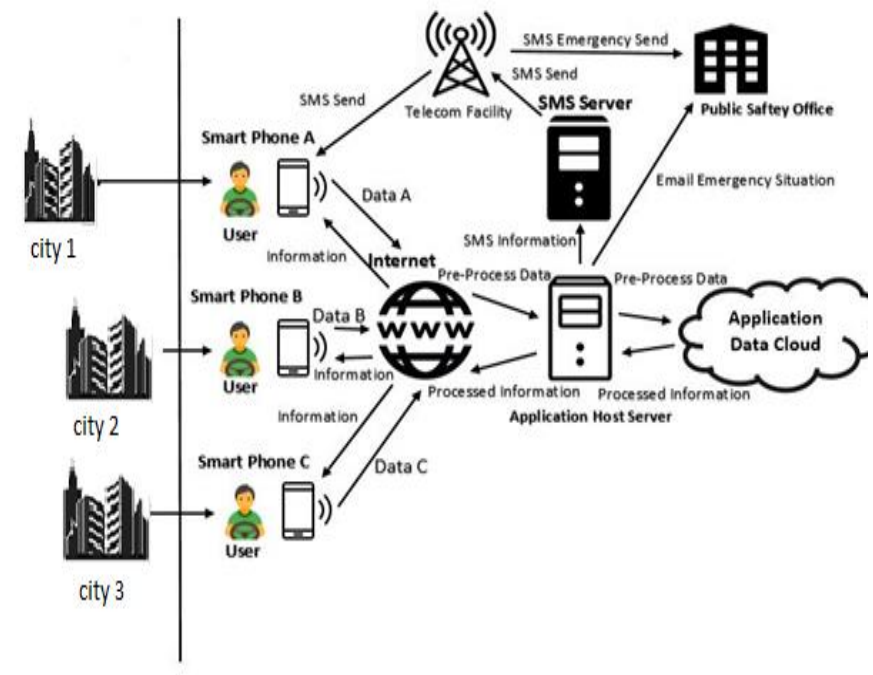

Fig. 3. Proposed Model of Smart City App.
Studies suggest that smart cities need specific information for experiencing globalization by making efficient smart city decisions like smart transportation, smart energy distribution, demo-graphic information, smart utilities, healthcare services, etc.

Travelers from one city to another city have very little information about nearby pinpoint spatial locations, safety organisations, emergency services and government building and necessary information for traveller and visitors with them. In case of any emergency situation, even local public safety organizations have no personal, medical records or emergency contact numbers for any situation. On any highways, peoples hesitate to do or accept any help from others travellers or unknown peoples because of no information. To overcome these issues with smart sensors, surveillance cameras, Wireless Fidelity (Wi-Fi) devices may help the citizen of smart-cities by the following critical technical innovations:

- Users will get real-time and dynamic information about the city routes with other cities in a particular range by Google Map as shown in Fig. 4. Smart mobile-app collected device and personal mobile information for notification, newsfeeds in text or audio format.

- Smart city will alert their citizens about road congestion in the form of text, audio/video format.

- Provide necessary information of other cities around and also provides a platform to communicate with these connected cities by text messages.

- Track record of smart cities user is or travellers from start to destination and generates alerts of essential places nearby like fuel stations, restaurants, hospitals, emergency stations, etc.

- Smart cities are real-data streaming analytics which provides complete details of their citizen with utility services to transportations facilities.

- Users can send and receive with one push any emergency messages to other cities users (using GSM/GPS, other Internet services) as well as inform nearby emergency organizations in the form of an email as shown in Fig. 5.

- Users can comfortably offer or accept emergency pickup, health-care services, nearby building info and share visits from other travellers especially on highways because smart cities will keep track of these connected cities.

- Reporting of any crime, security surveillance, weather forecast, misconduct to authorities nearby (if witnessed) with proper privacy.

- The user receives text messages as well as audio/videos format to prevent mental divergence. 


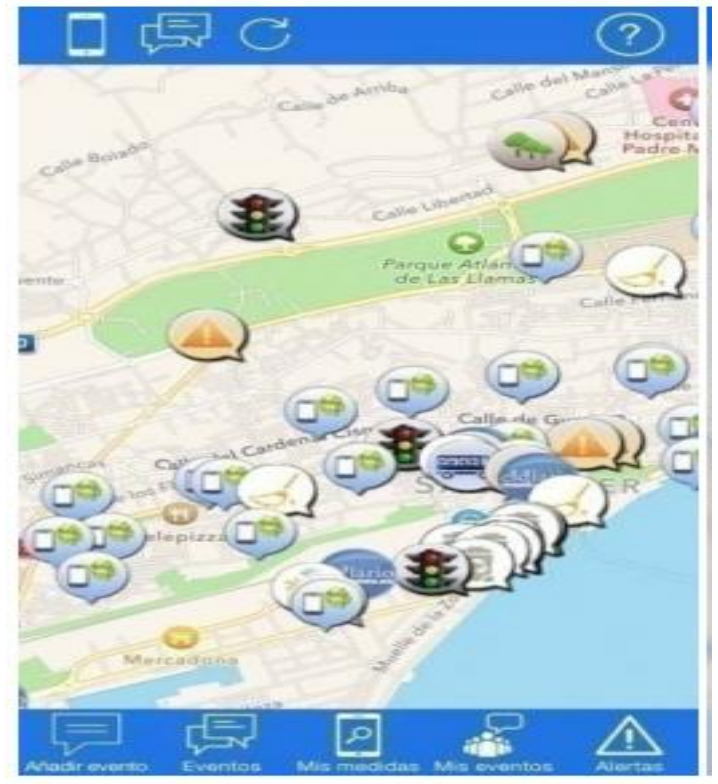

Fig. 4. Sample prototype of Smart-Map.

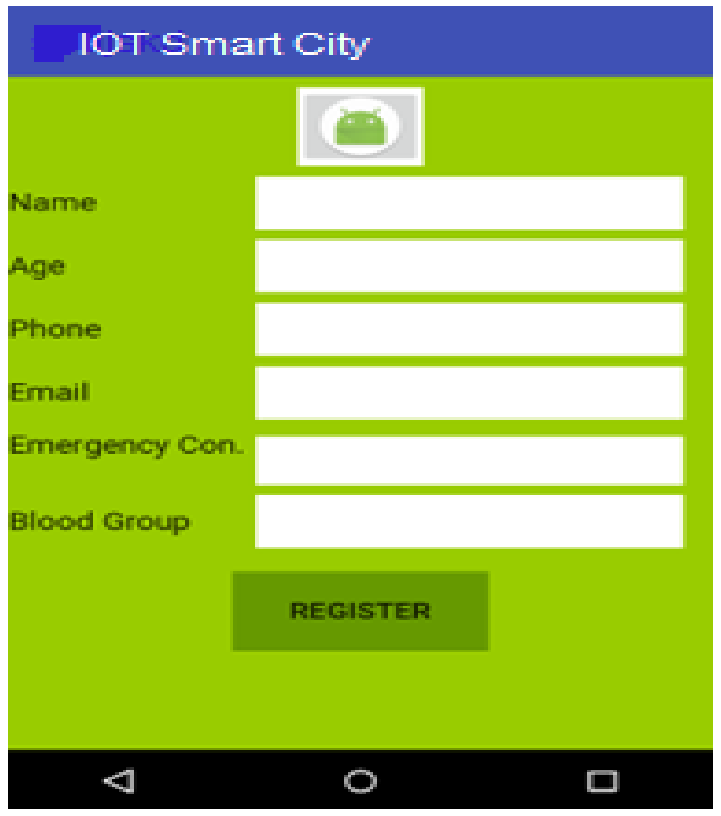

Fig. 5. Prototype of Smart-City App.

Smart cities users are using user's smartphone as sensing as well as a communication device. In the proposed system smartphones will act as wireless sensor network's node. Internet and Mobile telecommunication GSM signals will act a medium of communication between all wireless networked sensor nodes. The application server will host smart-cities application and is connected to Short Message Service (SMS) server which will generate text messages, and it is also an interface between end nodes and application cloud-server. The application server will also send e-mails to public safety organizations in case of an emergency. The application server will provide all the necessary computations. Microsoft Azure IoT cloud server will be used because of its enhanced features for smart cities utility services, transportation conditions, environmental conditions and security features realization.

\section{SOCIAL ANALYSES}

In this section social analysis of smart cities as a sample has been performed in the form of graphical representation. A graph is a data structure which consisting of a finite number of edges and nodes. There are many ways to represents a node, edge graph, for example, adjacency matrix, graph ML format, CSV files.

The adjacency matrix is a two-dimensional square matrix whose size is equal to the number of nodes in the graph. However, if input graph contains a large number of nodes and less number of edges then the adjacency matrix became sparse and space consuming. Fig. 6 represents a sample connected cities graph and Table I represents an adjacency matrix $6 * 6$ of connected cities.

In the graph illustrated in Fig. 6 nodes represent cities at different ranges and edges for instance roads between cities, paths and connectivity or relationship between cities are in different ranges.

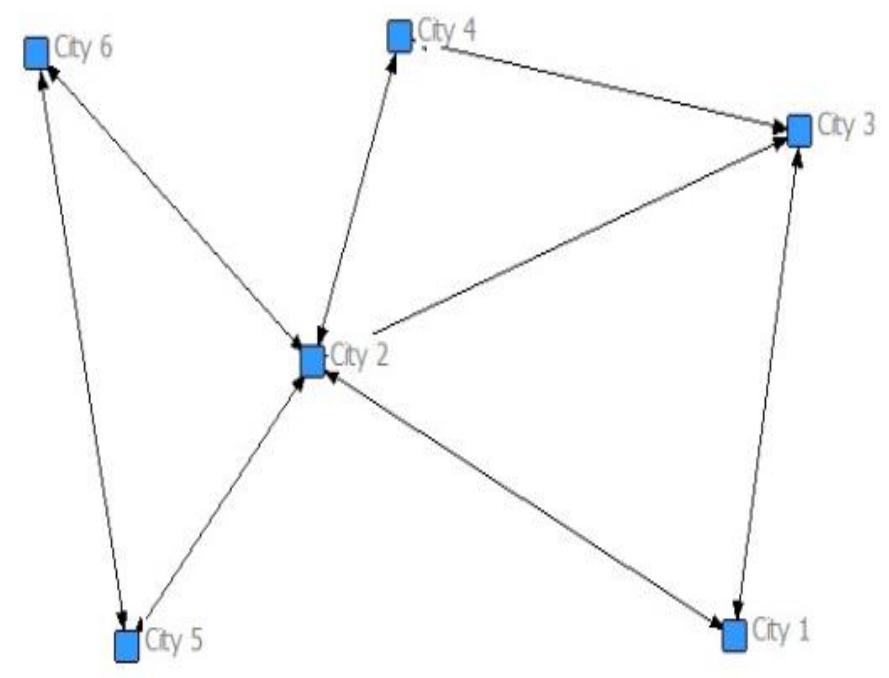

Fig. 6. Connected smart cities graph.

Edges define the relationship between different users or cities resources, a directed edge from city 1 to city 2 represents that city one can communicate with city 2 and city 3 . City 2 can communicate with city 5 , city 4 and city 3 and so on for every city connection in a graphical format having close centrality measures. The adjacency matrix is represented in Table I in this " 0 " represents no relationship, and " 1 " represents the positive relationship. If city one users want to communicate with city four user's, they can communicate with the help of city two based on shortest path algorithm between two nodes traversed. 
TABLE I. ADJACENCY MATRIX BETWEEN CITIES 6 X 6

\begin{tabular}{|l|l|l|l|l|l|l|}
\hline & City 1 & City 2 & City 3 & City 4 & City 5 & City 6 \\
\hline City 1 & 0 & 1 & 1 & 0 & 0 & 0 \\
\hline City 2 & 1 & 0 & 1 & 1 & 1 & 1 \\
\hline City 3 & 1 & 0 & 0 & 1 & 0 & 0 \\
\hline City 4 & 0 & 1 & 1 & 0 & 0 & 0 \\
\hline City 5 & 0 & 1 & 0 & 0 & 0 & 1 \\
\hline City 6 & 0 & 1 & 0 & 0 & 1 & 0 \\
\hline
\end{tabular}

This phenomenon is used on the higher level as well in computational intelligence. In which every node represents a smart city a cluster of cities and edges represents any one of cites which can reside or act as the interaction between two groups or clusters. The central city would be helpful for communication between cities in different geographical location city areas might be other cities. This technique will enhance the range of communication between two distanced cities. The model of communication of distant (out of the range) cites clusters shown in Fig. 7.

The adjacency matrix of connected cities is shown in Table II.

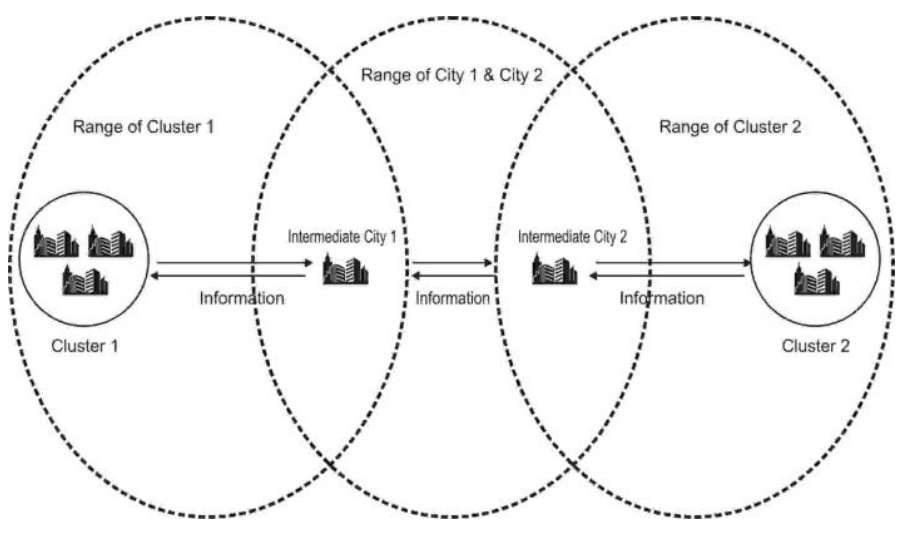

Fig. 7. Communication of cities clusters.

TABLE II. ADJACENCY Matrices of Distant Cities

\begin{tabular}{|l|l|l|l|l|}
\hline & Cluster 1 & $\begin{array}{l}\text { Inter } \\
\text { City1 }\end{array}$ & $\begin{array}{l}\text { InterCity } \\
\mathbf{2}\end{array}$ & Cluster 2 \\
\hline Cluster 1 & 0 & 1 & 0 & 0 \\
\hline $\begin{array}{l}\text { Intermediate } \\
\text { City1 }\end{array}$ & 1 & 0 & 1 & 0 \\
\hline $\begin{array}{l}\text { Intermediate } \\
\text { City2 }\end{array}$ & 0 & 1 & 0 & 1 \\
\hline Cluster 2 & 0 & 0 & 1 & 0 \\
\hline
\end{tabular}

\section{PROPOSED MFIS BASED SOLUTION}

This section explains in detail Mamdani Fuzzy Inference System (MFIS) based on smart-city Traffic Congestion Conditions (TCC) controls. The facts given below explain the measuring of TCC for the Smart-city for smart drive facility which is based on Mamdani Fuzzy logic principles.

In this article, the planned MFIS which is capable of measuring the TCC for the city algorithm is given in Table III. The five inputs and one output MFIS is proposed to calculate TCC.

In this method five inputs that are: Vehicle Speed $(\mathrm{S})$, Load Capacity (C), Traffic Signals (T), Distance between Signal (D), Riding Distance (R) are taken. These inputs are used to build up a lookup table given in Table IV to decide TCC for a respective algorithm for input-output relation given by MFIS. Its mathematical representation is shown in (1).

$$
\mu_{\mathrm{CG}}=\operatorname{MFIS}\left[\mu_{\mathrm{VS}}, \mu_{\mathrm{RD}}, \mu_{\mathrm{TS}}, \mu_{\mathrm{DTS}}, \mu_{\mathrm{RD}}\right]
$$

In this article, the Intelligent Transportation System (ITS) is measured using Mamdani Fuzzy Inference System (MFIS). Table I shows the proposed MFIS Based ITS algorithm. The I/O surface for MFIS is given in Fig. 1.

TABLE III. PROPOSED MFIS BASED TCC ALgORITHM

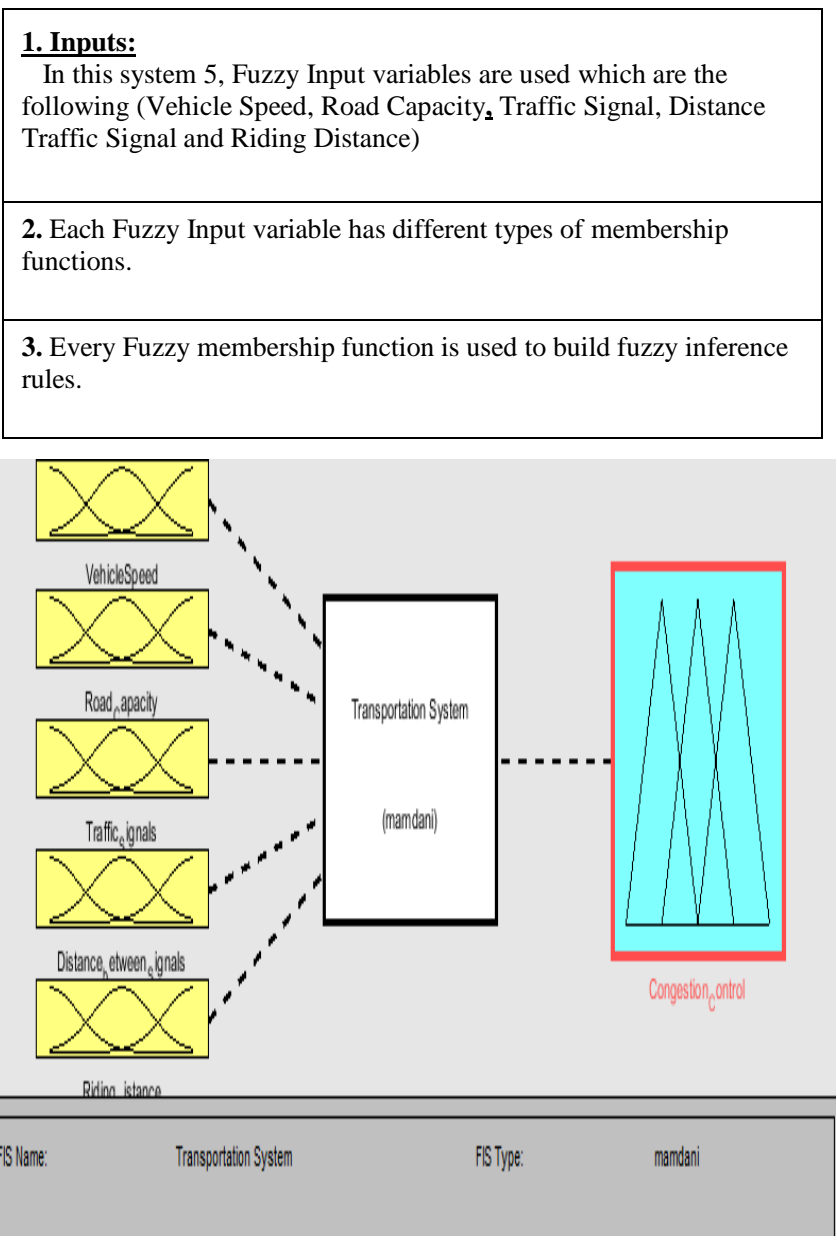

Fig. 8. Input and output surface for MFIS. 
TABLE IV. MATHEMATICAL AND GRAPHICAL MF OF MFIS INPUT VARIABLES

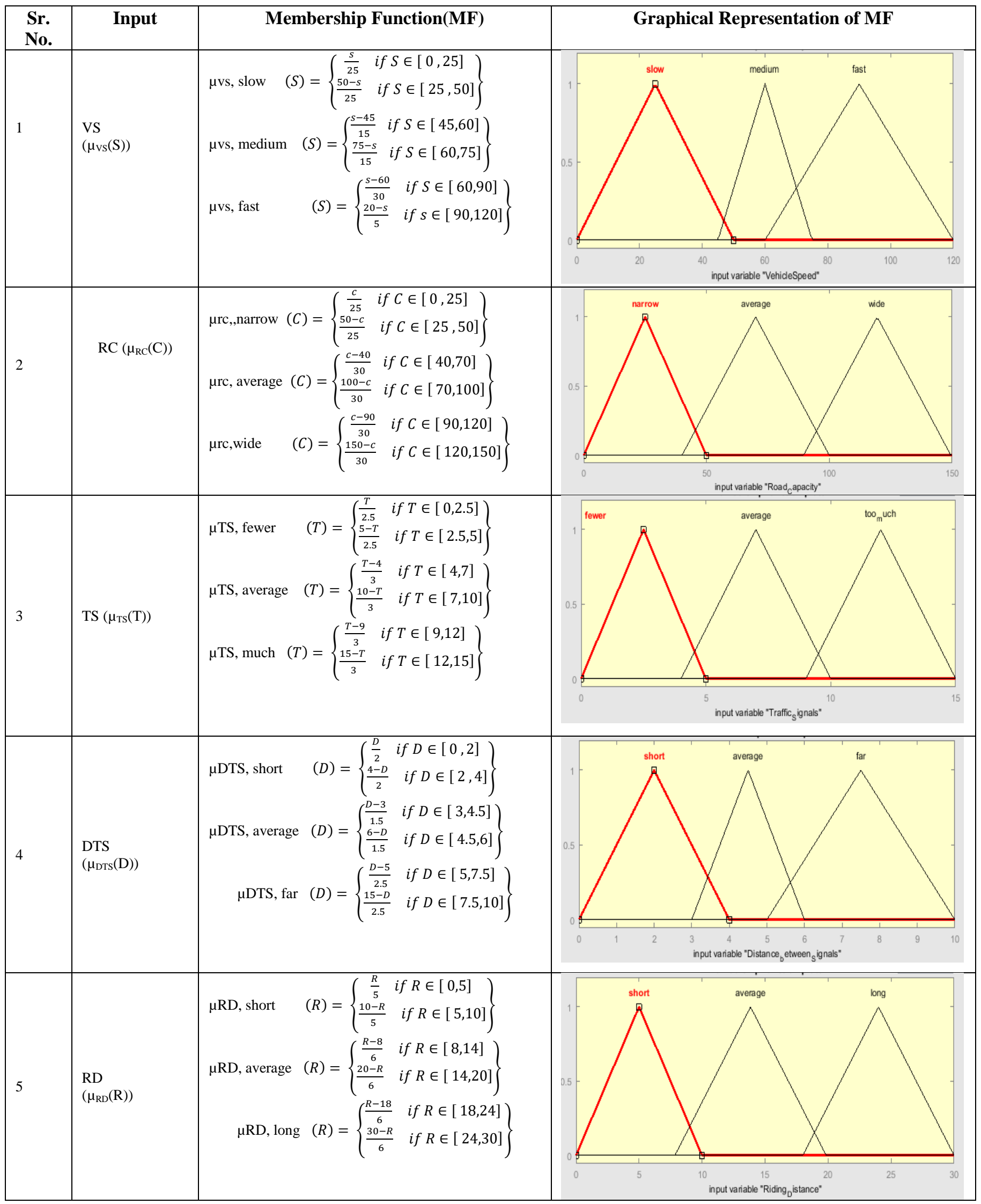


TABLE V. INPUT VARIABLE RANGES

\begin{tabular}{|l|l|l|l|}
\hline Sr \# & $\begin{array}{l}\text { Input } \\
\text { Parameters }\end{array}$ & Ranges & Semantic sign \\
\hline \multirow{3}{*}{1} & VS & $0-50$ & Slow \\
& & $45-75$ & Medium \\
& $60-120$ & Fast \\
\hline \multirow{3}{*}{2} & Cap & $0-50$ & Narrow \\
& & $40-100$ & Average \\
& & $90-150$ & Wide \\
\hline 3 & TS & $0-5$ & Fewer \\
& & $4-10$ & Normal \\
\hline & & $9-15$ & Too much \\
\hline 4 & DTS & $2-4$ & Nearer \\
& & $3-6$ & Average \\
\hline \multirow{3}{*}{5} & RD & $5-10$ & Far \\
\hline & & $0-10$ & Nearer \\
& & $18-20$ & Center \\
\hline
\end{tabular}

\section{A. Input Fuzzy Sets}

Fuzzy input variable is statistical values that are used to calculate the Traffic Congestion Condition in smart cities.

In this article, five different types of fuzzy variables are used for the analysis of congestion in smart cities. The detail of these input variables is given in Table V.

\section{B. Fuzzy Output Variable}

Fuzzy output variable Traffic Congestion Control (TCC) is used to calculate the result by the values of input variables in the world of discourse. The details of output are shown in Table VI.

TABLE VI. OUTPUT VARIABLE RANGES

\begin{tabular}{|l|l|l|l|}
\hline Sr \# & Output of MFIS & Ranges & $\begin{array}{l}\text { Semantic sign for } \\
\text { Congestion }\end{array}$ \\
\hline 1 & Congestion Control & $\begin{array}{l}0-0.5 \\
0.2-0.7 \\
0.5-1\end{array}$ & $\begin{array}{l}\text { No delay (Less) } \\
\text { Average delay } \\
\text { (Medium) } \\
\text { Much delay (High) }\end{array}$ \\
\hline
\end{tabular}

\section{Membership Functions}

Membership function gives curve value between 0 and 1, and it provides a mathematical function which provides statistical values of input and output variable. Membership functions are also available in MATLAB tool. The propose solution uses the membership function which is as follows:

- Trim

Trim is a triangular curve built-in MATLAB function. To calculation of this function, three scalar parameters are used in the proposed solution which is Low, Medium, and High. The mathematical equations and graphical representation of membership function are given in Table IV.

\section{Rule-Based}

In this system most, suitable rules for system understanding are applied. This rule-base system contains about 81 input-output rules, the system complexity increased if the number of rules increased. The Mamdani Fuzzy Inference rules are shown in Fig. 9.

\section{E. Inference Engine}

The Mamdani Inference Engine is used to map five inputs to one output (TCC) as shown in Fig. 8.

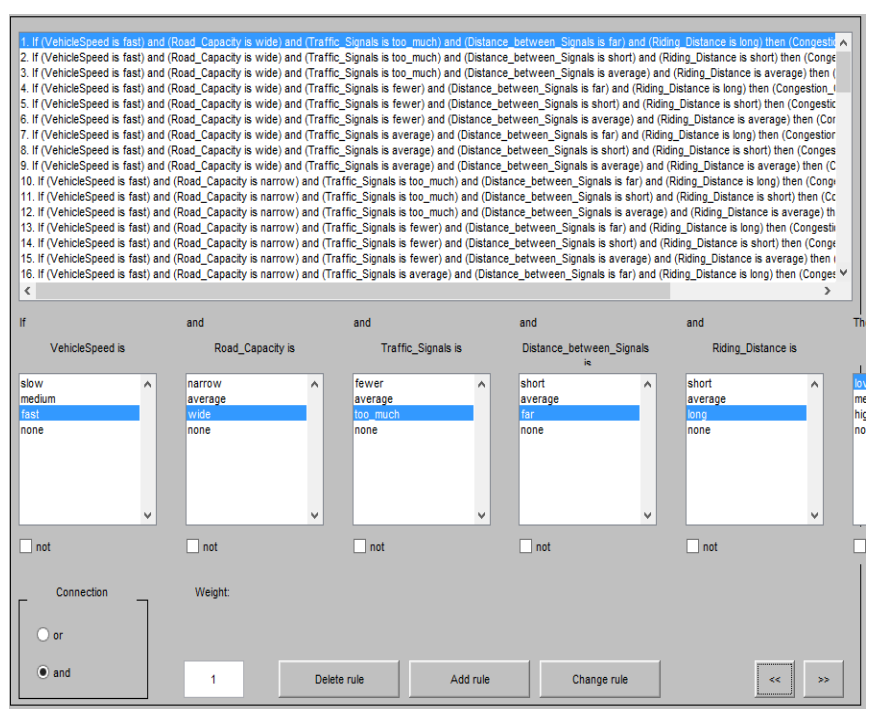

Fig. 9. I/O Rules for ITS.

\section{F. De-Fuzzifier}

In this article centroid, De- Fuzzifier is used. Fig. 10 to 12 represents rule surface of Proposed ITS using MFIS.

Fig. 10 shows that if Vehicle Speed is between $1-80 \mathrm{~km} / \mathrm{s}$ and Traffic Signals are lies in the range of 10 to 15 , then Traffic Congestion is approximately $80 \%$, which is high.it also shown that, if Vehicular Speed between $80-120 \mathrm{~km} / \mathrm{s}$ and Traffic Signal is 10-15, then Congestion is low approximately $10 \%$.

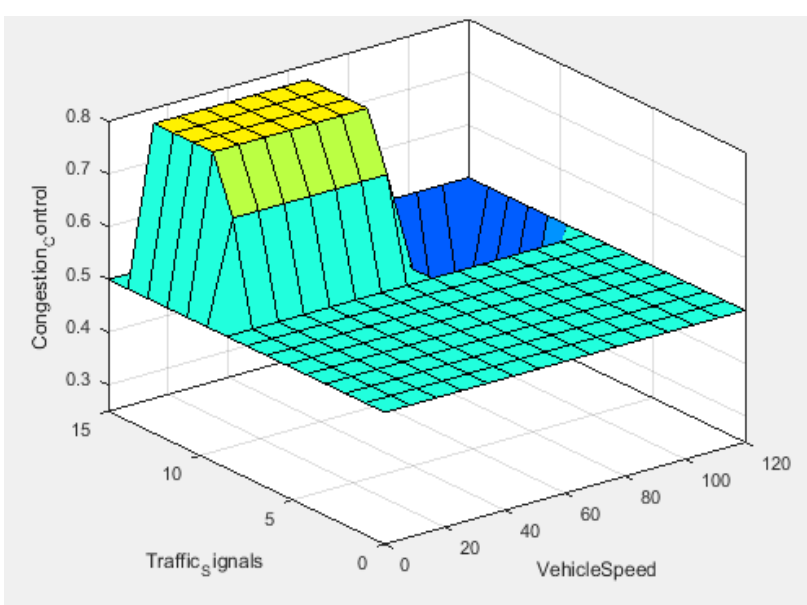

Fig. 10. Rule surface for traffic signals and vehicle speed.

Fig. 11 shows Congestion Control using input variables Traffic signals and Riding Distance. It is observed that congestion is approximately $80 \%$ when Traffic signals are 1015 and Riding Distance between Source to Destination lies in 
the range of 8 to $20 \mathrm{~km}$. Congestion is approximately $60 \%$ if Traffic signals are 9-10 and Riding Distance grater then $8 \mathrm{~km}$.

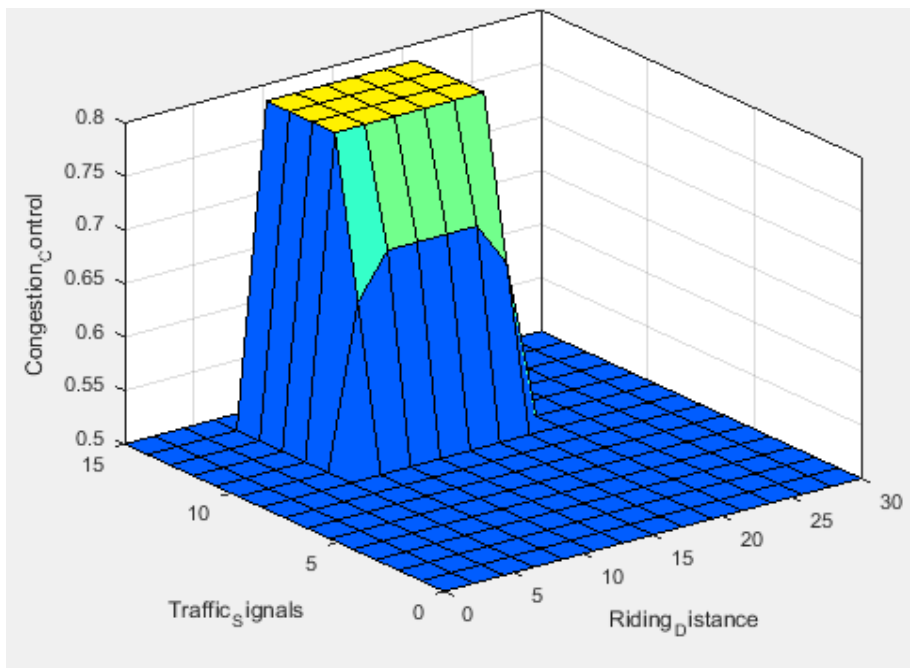

Fig. 11. Rule surface for traffic signals and riding distance.

Fig. 12 shows Congestion depends upon input variables Road Capacity and Riding Distance between source to destination. Approximately, there is no Congestion when road capacity is extensive (110 to 120 vehicles on the road) and Riding Distance is 9 to $20 \mathrm{~km}$. If Road Capacity between 100 to 110 approx and Riding Distance between 10 to $20 \mathrm{~km}$ then congestion is increased upto $20 \%$ increase. If road capacity is less than 90 (narrow road), then the congestion is up to $50 \%$.

So, it concludes that Congestion inversely proportional to Road Capacity.

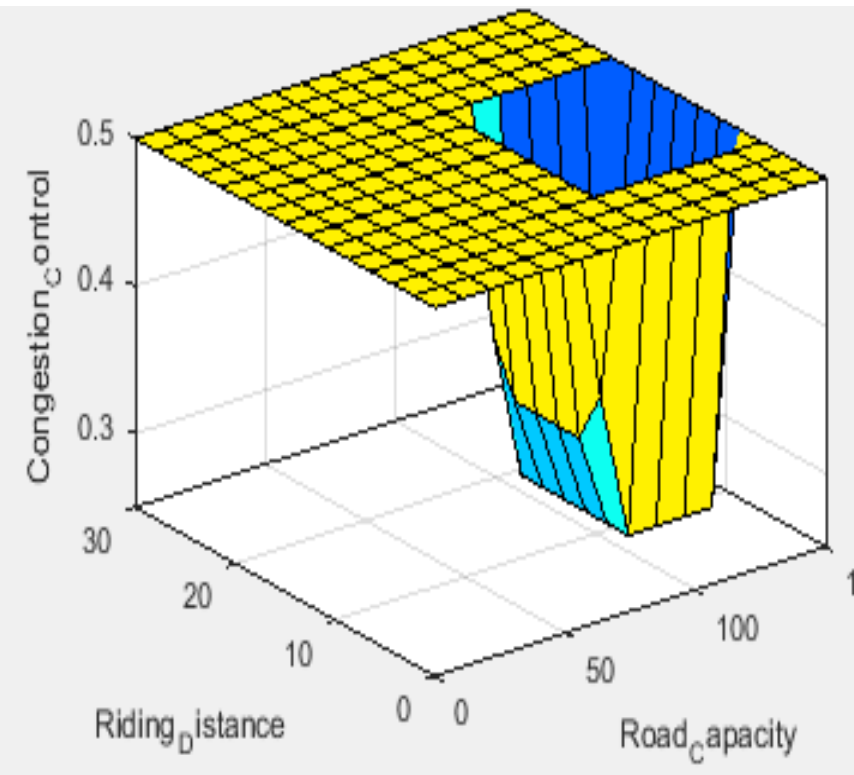

Fig. 12. Rule surface for riding distance and road capacity.

\section{G. Simulation Results}

For simulation results, MATLAB R2017a tool is used. MATLAB is also used for modelling, simulation, algorithm development, prototyping and many other fields. MATLAB is an efficient tool for programming, data analysis, visualisation and computing. For simulation results, five inputs and one output Congestion Control variable is used.

TABLE VII. CONGESTION CONTROL BASED ON RULES DEFINED

\begin{tabular}{|l|l|l|l|l|l|}
\hline \multicolumn{4}{|l|}{ Input Variables } & Output \\
\hline VS & RC & TS & DTS & RD & Congestion \\
\hline H & W & L & H & H & Less Delay \\
\hline H & N & H & L & L & Medium Delay \\
\hline L & N & H & L & L & High Delay \\
\hline
\end{tabular}

Table VII explains the rules of Proposed Congestion Control system. Fig. 13 to 15 shows the proposed system evaluation.

Fig. 13 shows the congestion is less if vehicular speed is high and road capacity is wide. It further depicts that if a traffic signal is few and distance between signals is high and riding distance is far congestion is low.

Fig. 14 shows that congestion is Medium if the vehicle speed is high and Road Capacity is Narrow, and traffic signals are too much, and the distance between signals is low, and riding distance is far then congestion is medium.

Fig. 15 explains congestion is high if the vehicle speed is Low and Road Capacity is Narrow, and traffic signals are too much, and the distance between signals is small, and riding distance is also low than congestion is high.

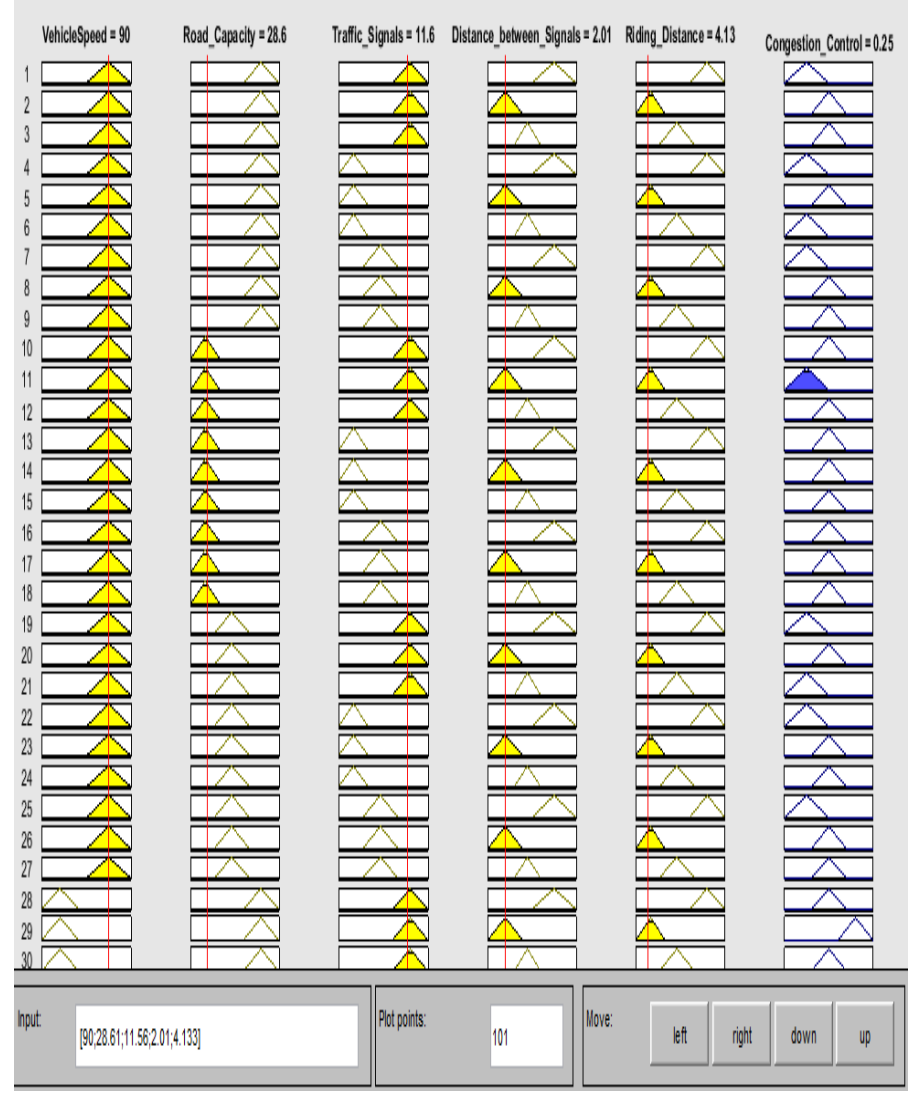

Fig. 13. Lookup diagram for low TCC. 


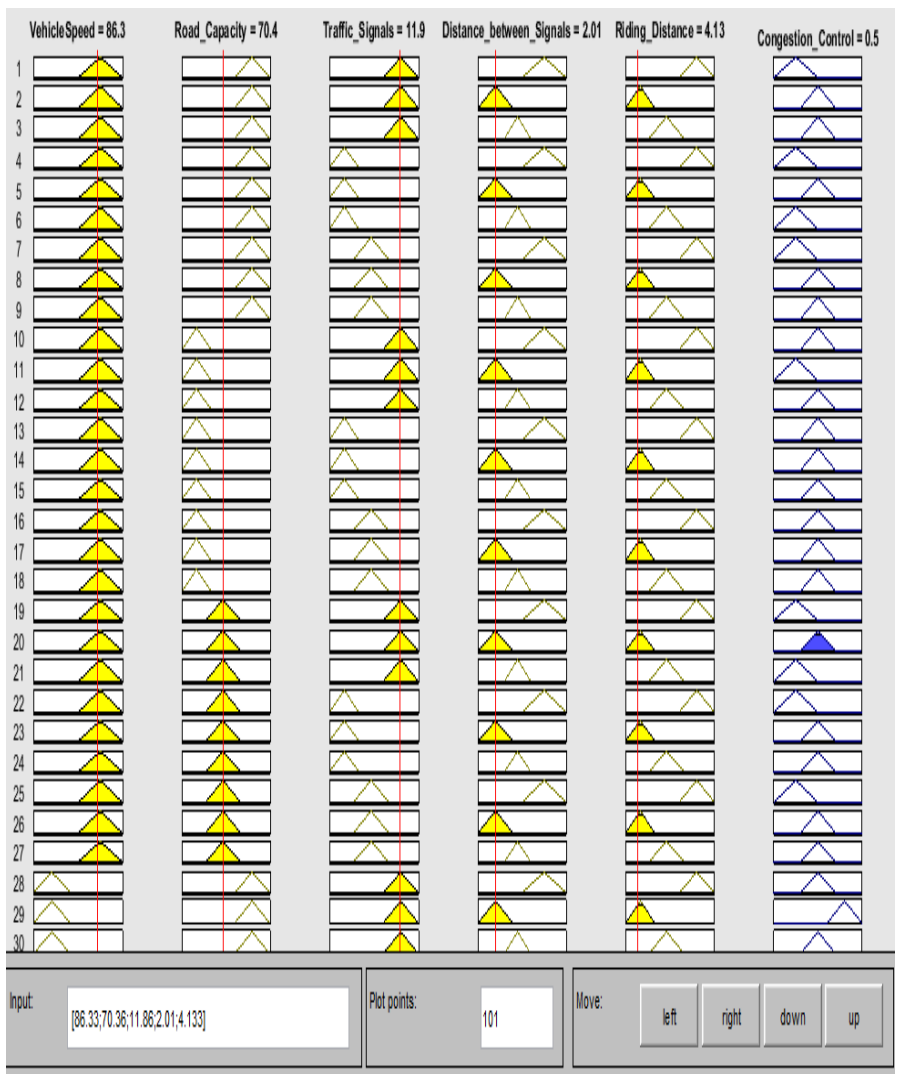

Fig. 14. Lookup diagram for medium TCC.

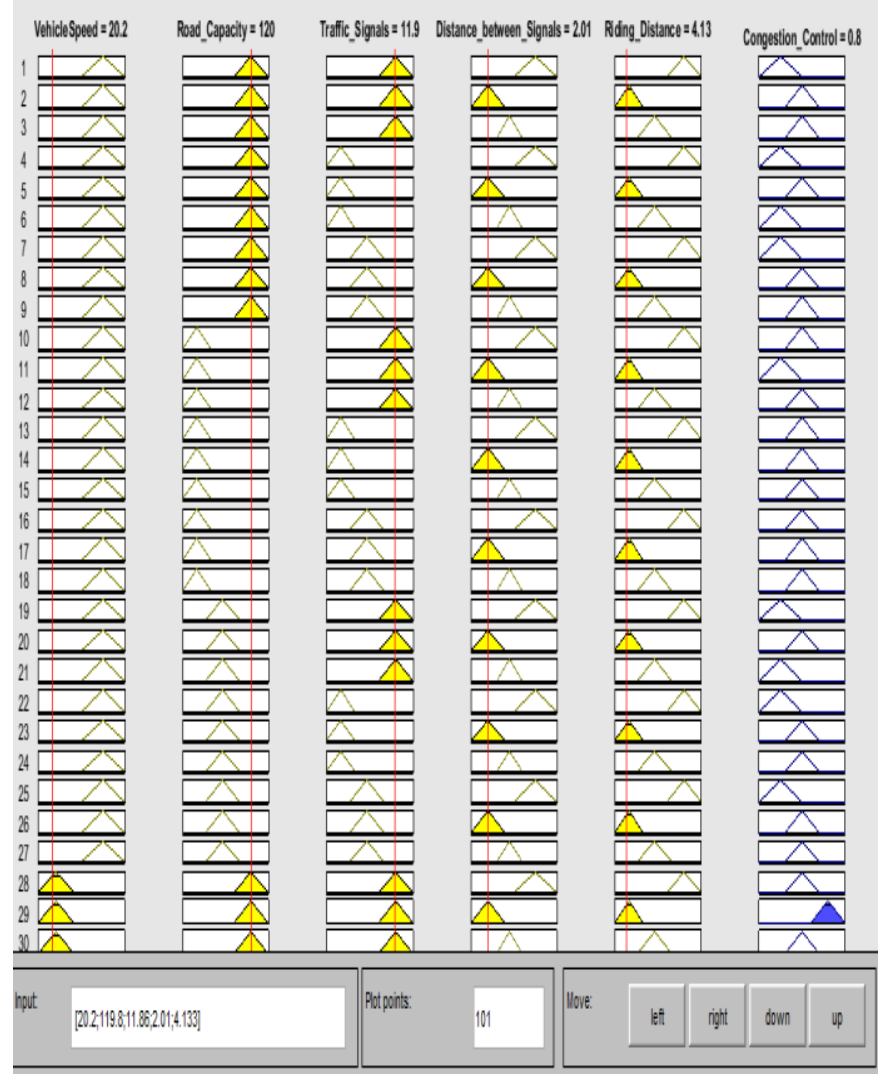

Fig. 15. Lookup diagram for high TCC.

\section{CONCLUSION AND FUTURE WORK}

Implementation of smart-cities infrastructure and services is a long-game process [25]. The advantages of smart-city to communities will not likely be quick and will probably be incremental in the first step. Nevertheless, to accomplish smart-cities infrastructure through the utilization of SNA, Information Communication Technologies and IoT's to scale their city framework and extend services reasonably while offering substantial financial advantages. This TCC fuzzy expert system is designed with the help of 5 input and one output variable. Mamdani Fuzzy Inference System (MFIS) is used to evaluate the Traffic Congestion Conditions (TCC) in smart-city. The proposed system design of TCC has been beneficial to determine the city traffic congestion. Through this system, anybody can check any traffic congestion. In future, MFIS would be used to evaluate the performance of the other resources of smart-city like Environmental Conditions, Energy Consumption, Healthcare and Security Surveillance, etc.

\section{REFERENCES}

1. Han, Q., Liang, S., \& Zhang, H. (2015). Mobile cloud sensing, big data, and $5 \mathrm{~g}$ networks make an intelligent and smart world. IEEE Network, 29(2), 40-45.

2. https://news.un.org/en/story/2014/07/472752-more-half-worldspopulation-now-living-urban-areas-un-survey-finds.

3. Carmona, Matthew. Public places, urban spaces: the dimensions of urban design. Routledge, 2010.

4. Kitchin, Rob. "Big Data, new epistemologies and paradigm shifts." Big Data \& Society 1.1 (2014): 2053951714528481.

5. Misuraca, Gianluca, Francesco Mureddu, and David Osimo. "Policymaking 2.0: Unleashing the power of big data for public governance." Open government. Springer, New York, NY, 2014. 171188.

6. Albino, Vito, Umberto Berardi, and Rosa Maria Dangelico. "Smart cities: Definitions, dimensions, performance, and initiatives." Journal of Urban Technology 22.1 (2015): 3-21.

7. Gabri Malek, Chunlin LI, Z. Yang, NajiHasan.A.H and X.Zhang,' Improved the Energy of Ad hoc On-Demand Distance Vector Routing Protocol', International Conference on Future Computer Supported Education, Published by Elsevier, IERI, pp. 355-361,2012.

8. Lakshmi, I. "A literature survey on Big Data Analytics in Service Industry." International Journal of Engineering and Computer Science 5.4 (2016). Breslin, J., Decker, S., 2007.

9. Dimitrakopoulos, George, and George Bravos. Current Technologies in Vehicular Communication. Springer, 2016.

10. Psomakelis12, Evangelos, et al. "BIG IOT AND SOCIAL NETWORKING DATA FOR SMART CITIES."

11. Nasr, Elie, Elie Kfoury, and David Khoury. "An IoT approach to vehicle accident detection, reporting, and navigation." In Multidisciplinary Conference on Engineering Technology (IMCET), IEEE International, pp. 231-236. IEEE, 2016

12. Rizwan, Patan, K. Suresh, and M. Rajasekhara Babu. "Real-time smart traffic management system for smart cities by using the Internet of Things and big data." In Emerging Technological Trends (ICETT), International Conference on, pp. 1-7. IEEE, 2016.

13. Al-Dweik, Arafat, Radu Muresan, Matthew Mayhew, and Mark Lieberman. "IoT-based multifunctional Scalable real-time Enhanced Road Side Unit for Intelligent Transportation Systems." In Electrical and Computer Engineering (CCECE), 2017 IEEE 30th Canadian Conference on, pp. 1-6. IEEE, 2017.

14. Goggin, Gerard. "Driving the internet: mobile internets, cars, and the social." Future Internet 4, no. 1 (2012): 306-321.

15. Joshi, Yashashree, Ashwini Joshi, Neha Tayade, Priyanka Shinde, and S. M. Rokade. "IoT Based Smart Traffic Density Alarming Indicator." 
(2016).Handte, Marcus, Stefan Foell, Stephan Wagner, Gerd Kortuem, and Pedro José Marrón. "An Internet-of-Things Enabled Connected Navigation System for Urban Bus Riders." IEEE Internet of things journal 3, no. 5 (2016): 735-744

16. Theodoridis, Evangelos, Georgios Mylonas, and Ioannis Chatzigiannakis. "Developing an IoT smart city framework." In Information, intelligence, systems and applications (Lisa), 2013 fourth international conference on, pp. 1-6. IEEE, 2013.

17. Chong, Hon Fong, and Danny Wee Kiat Ng. "Development of IoT device for traffic management system." In Research and Development (SCOReD), 2016 IEEE Student Conference on, pp. 1-6. IEEE, 2016

18. Chong, Hon Fong, and Danny Wee Kiat Ng. "Development of IoT device for traffic management system." In Research and Development (SCOReD), 2016 IEEE Student Conference on, pp. 1-6. IEEE, 2016

19. Thakur, Tanvi Tushar, Ameya Naik, Sheetal Vasari, and Manjiri Gogate. "Real-time traffic management using the Internet of Things." In Communication and Signal Processing (ICCSP), 2016 International Conference on, pp. 1950-1953. IEEE, 2016

20. Ramachandra, Sujit H., K. Nitesh Reddy, Vivek R. Vellore, Sumanth Karanth, and Tareesh Kamath. "A novel dynamic traffic management system using on-board diagnostics and Zigbee protocol."
21. In Communication and Electronics Systems (ICCES), International Conference on, pp. 1-6. IEEE, 2016

22. Chowdhury, Abdullahi. "Priority-based and secured traffic management system for an emergency vehicle using IoT." In Engineering \& MIS (ICE), International Conference on, pp. 1-6. IEEE, 2016

23. Ou, Haoyuan, Jianming Zhang, and Yi Wang. "Development of intelligent traffic control system based on internet of things and fpga technology in proteus." traffic 20 (2016): 2.

24. Amadeo, Marica, Claudia Campolo, Jose Quevedo, Daniel Corujo, Antonella Molinaro, Antonio Iera, Rui L. Aguiar, and Athanasios V. Vasilakos. "Information-centric networking for the internet of things: challenges and opportunities." IEEE Network 30, no. 2 (2016): 92-100.

25. Coutard, Olivier, et al. "Urban Megatrends: Towards a European research agenda." (2014): 1-17.

26. Saeed, Yousaf, et al. "Impact of Cognition on User Authentication Scheme in Vehicle using Fuzzy Logic and Artificial Neural Network." International Journal of Computer Science and Information Security 14.10 (2016): 285.

27. Abbas, Sagheer, et al. "Bio-inspired neuro-fuzzy based dynamic route selection to avoid traffic congestion." International Journal of Scientific and Engineering Research2.6 (2011): 284-289. 